\title{
Pathologic Characteristics Associated With Local Recurrence of Atypical Meningiomas Following Surgical Resection
}

\author{
Laura Zima ${ }^{\mathrm{a}, \mathrm{f}}$, Michael J. Baine ${ }^{\mathrm{b}, \mathrm{f}}$, Richard Sleightholm ${ }^{\mathrm{c}}$, Bangchen Wang ${ }^{\mathrm{c}}$, \\ Michael Punsoni ${ }^{\mathrm{d}}$, Michele Aizenberge ${ }^{\mathrm{e}}$, Chi Zhang,
}

\begin{abstract}
Background: Optimal management of grade II meningiomas following resection remains controversial, owing mostly to the heterogeneity of post-operative (post-op) recurrence patterns across studies. Improved risk stratification of these patients would ensure that only those most at risk of recurrence would undergo appropriate post-op radiation therapy $(\mathrm{RT})$.
\end{abstract}

Methods: Medical records from patients who underwent resection for grade II meningiomas were retrospectively reviewed. Demographic, disease characteristics, treatment, and clinical course data were retrospectively collected. Logistic regression, Cox proportional hazards modeling, and Kaplan-Meier curves with log rank testing were conducted to describe any potential relationships with time of recurrence.

Results: Of the 49 patients identified, 18 (36.7\%) suffered a local recurrence following resection with a median follow-up of 3.1 years (range: 0.23 - 17.1 years). Past recurrence of the meningioma $(\mathrm{P}=$ $0.002)$ and extent of resection $(\mathrm{P}=0.02)$ were significantly associated with local recurrence. On multivariable analysis, only prior meningioma recurrence was associated with time to local failure $(\mathrm{P}=0.021)$. No histopathologic factors were found to be associated with the initial local failure. Of those who suffered a local recurrence, the presence of bone invasion (hazard ratio: $0.069, \mathrm{P}=0.008$ ) and lack of salvage RT

Manuscript submitted February 9, 2021, accepted February 23, 2021

Published online March 19, 2021

aDepartment of Neurosurgery, University of Texas Health Science Center at Houston, Houston, TX, USA

bDepartment of Radiation Oncology, Fred and Pamela Buffett Cancer Center, University of Nebraska Medical Center, Omaha, NE, USA

${ }^{\mathrm{c}}$ College of Medicine, University of Nebraska Medical Center, Omaha, NE, USA

${ }^{\mathrm{d}}$ Department of Pathology, University of Nebraska Medical Center, Omaha, NE, USA

eDepartment of Neurosurgery, University of Nebraska Medical Center, Omaha, NE, USA

${ }^{\mathrm{f}}$ These authors contributed equally to this article.

'Corresponding Author: Chi Zhang, Department of Radiation Oncology, University of Nebraska Medical Center, Buffett Cancer Center, 505 S 45th St, Omaha, NE 68106, USA. Email: chi.zhang@unmc.edu

doi: https://doi.org/10.14740/jocmr4444
$(\mathrm{P}=0.02)$ were associated with subsequent local failure.

Conclusions: Currently considered histopathologic factors appear not to be helpful in guiding initial treatment course. History of prior local failure and bone invasion appear to be associated with multiple recurrences. Optimal surgical resection is critical to improving outcomes, and salvage RT may reduce subsequent local failure.

Keywords: Grade II meningioma; Gross/subtotal resection; Radiation therapy; Predictive markers

\section{Introduction}

Meningiomas are one of the most common types of primary intracranial neoplasms. They are grouped into World Health Organization (WHO) grades I, II, and III [1]. The majority, $80-90 \%$, of meningiomas are classified as grade I [2]. These are typically benign and defined by $<4$ mitoses per 10 highpowered fields (HPF) [1]. A small percentage, 1-3\%, of meningiomas are classified as grade III [2]. These are anaplastic and aggressive. They are defined by $>20$ mitoses per $10 \mathrm{HPF}$ or frank carcinomatous/sarcomatous histology [1]. The remaining $5-15 \%$ of meningiomas are classified as grade II, with criteria including having 4 - 19 mitoses per HPF, brain invasion, or three of the following five histologic features: increased cellularity, small cells with high nuclear/cytoplasmic ratio, large and prominent nucleoli, sheet-like growth, and/or foci of necrosis $[1,2]$. Grade II meningiomas are a more heterogeneous group than grades I and III, with higher intergroup variability in cellular characteristics and behavior.

Gross total resection (GTR) of a grade I meningioma is generally curative, and post-operative radiation is standard after GTR of a type III meningioma due to high propensity for recurrence. Optimal management of grade II meningiomas following GTR remains controversial, owing mostly to the heterogeneity of post-operative recurrence patterns across studies [3]. Recurrence of type II meningiomas after resection varies from $20 \%$ to $70 \%[2,4,5]$. Improved risk stratification of these patients would help to ensure that only those patients most at risk of recurrence would be selected to undergo appropriate post-operative radiation therapy (RT). 
A prime example of this heterogeneity in aggressiveness in grade II meningiomas comes from the recently reported NRG Oncology RTOG 0539 phase II clinic trial. In this study, patients with intermediate risk meningiomas, defined as newly diagnosed WHO grade II with GTR (Simpson grade I - III, $69.2 \%$ of enrolled patients) or recurrent WHO grade I with any resection grade $(30.8 \%$ of enrolled patients) were treated with adjuvant RT and compared to historical controls who did not receive radiation [6]. Initial results from this study showed that patients treated with RT after GTR had improved 3-year progression-free survival compared to those who just had GTR, $90 \%$ vs. $70 \%$ respectively. While this data are promising in that it demonstrates the efficacy of RT in this patient population, it is notable that only $30 \%$ of these patients would progress without radiation. This consideration begs the question of whether it is reasonable to irradiate all patients with grade II meningiomas to reduce the $30 \%$ of patients who may recur by two-thirds. Alternatively, differentiating which grade II patients are more likely to recur is crucial to providing more optimal treatment recommendations that will maximize patient benefit and minimize over-treatment.

Currently, it remains unclear which factors are associated with post-operative recurrence in this patient population. To date, multiple groups have attempted to determine histologic, physiologic, and anatomic determinants of aggressiveness, with specific factors studied including Simpson grade, Shinshu grade, location, different radiographic features including edema, calcification, and bone invasion, and Ki-67 among others $[4,7,8]$. However, several of these studies have inconsistent findings such as Ki-67 being found to be predictive of recurrence by multiple institutions while others have not found it to be significant $[4,8-12]$.

More research is needed to determine which pathologic and patient features, if any, are predictive of recurrence in grade II meningiomas as such information would guide postoperative RT recommendations. Our study seeks to further delineate the predictive nature of patient and pathologic features in grade II meningiomas. Our hypothesis is that certain features or combinations there-of will correlate with recurrence and outcomes in grade II meningiomas and thus help to predict tumor behavior.

\section{Materials and Methods}

This study was approved and conducted in ethical compliance by the Institutional Review Board at the University of Nebraska Medical Center (UNMC), which holds its standards to the highest standards such as those presented forth by the Helsinki Declaration on human research. A retrospective chart review of patients who had pathologically proven grade II meningiomas and were treated at UNMC was conducted. All pathologic specimens were reviewed by a neuropathologist in the Pathology Department at UNMC. Data were retrospectively collected from the electronic health records and included: demographic information, extent of resection, histopathologic features, clinical course, and treatment and recurrence history. Specific pathologic characteristics that were analyzed included: number of mitoses per HPF, presence or absence of prominent nucleoli, architectural sheeting, hypercellularity, choroid or clear cell histology, necrosis, and bone/brain invasion as well as Ki-67. Extent of resection was determined from the operative report and confirmed by review of post-operative imaging. Simpson grading was not collected due to inconsistent reporting in the post-operative documentation. Date of progression of meningioma was determined by radiology reports. For patients who were referred to our institution after suffering local recurrence following initial treatment at outside institutions, time of surgery at our institution was considered time zero for assessing time to subsequent local failure.

Logistical regression modeling was used to preliminarily describe any demographic or histologic features potentially associated with those who did or did not experience disease recurrence. Subsequent Cox proportional hazards $(\mathrm{CPH})$ modeling was conducted to further investigate the relationship of each factor as it related to time of recurrence. For variables found to be of interest, time to recurrence was visualized using Kaplan-Meier plots and the strength of correlation assessed using log-rank testing. $\mathrm{P}$ values $<0.05$ were considered statistically significant and $<0.10$ were considered borderline significant. For each statistical analysis, factors that were identified as being statistically significant were entered into a multivariable model to adjust for potential confounding effects.

\section{Results}

A total of 49 patients met inclusion criteria and were included in data analysis. Gender distribution was almost equal with 21 males and 28 females. Median age was 50 (range: 17 -90). Racial distribution was predominately white. Eighteen patients $(36.7 \%$ ) underwent a subtotal resection (STR), while 31 patients $(63.3 \%)$ underwent a gross total resection (GTR). Notably, 12 patients $(24.5 \%)$ underwent surgical resection at our institution after local recurrence following initial treatment at outside institutions. Eighteen patients (36.7\%) suffered a local recurrence following resection with a median follow-up after surgery of 3.1 years (range: $0.23-17.1$ years, Table 1 ).

A lower percentage of recurrence was observed in those who had a GTR compared to the STR cohort, 25.8\% vs. $55.6 \%$, respectively $(\mathrm{P}=0.064$, Table 1$)$. Further assessment of time to recurrence as assessed by Kaplan-Meier was associated with prolonged progression-free survival in the GTR cohort, $80 \%$ vs. 36\% 3-year progression-free survival $(\mathrm{P}=$ 0.010 , Fig. 1a). Interestingly, however, in patients who suffered recurrence, extent of resection displayed similar time to failure rates $(\mathrm{P}=0.176$, Fig. $1 \mathrm{~b})$; this was particularly true within the first year, and limiting a sub-analysis to time after the first year, there was a trend to prolonged progression-free survival in the GTR cohort compared to STR $(P=0.09)$. Importantly, patients who were referred to our institution after local failure following treatment at an outside facility were also more likely to suffer a subsequent recurrence than those who had not previously been treated for their meningioma (Table 1, Fig. 2). No other factors, including Ki-67 or the use of adjuvant RT, were significantly associated with local fail- 
Table 1. Patient Demographics and Features Predictive of Local Recurrence After Resection for Grade II Meningiomas

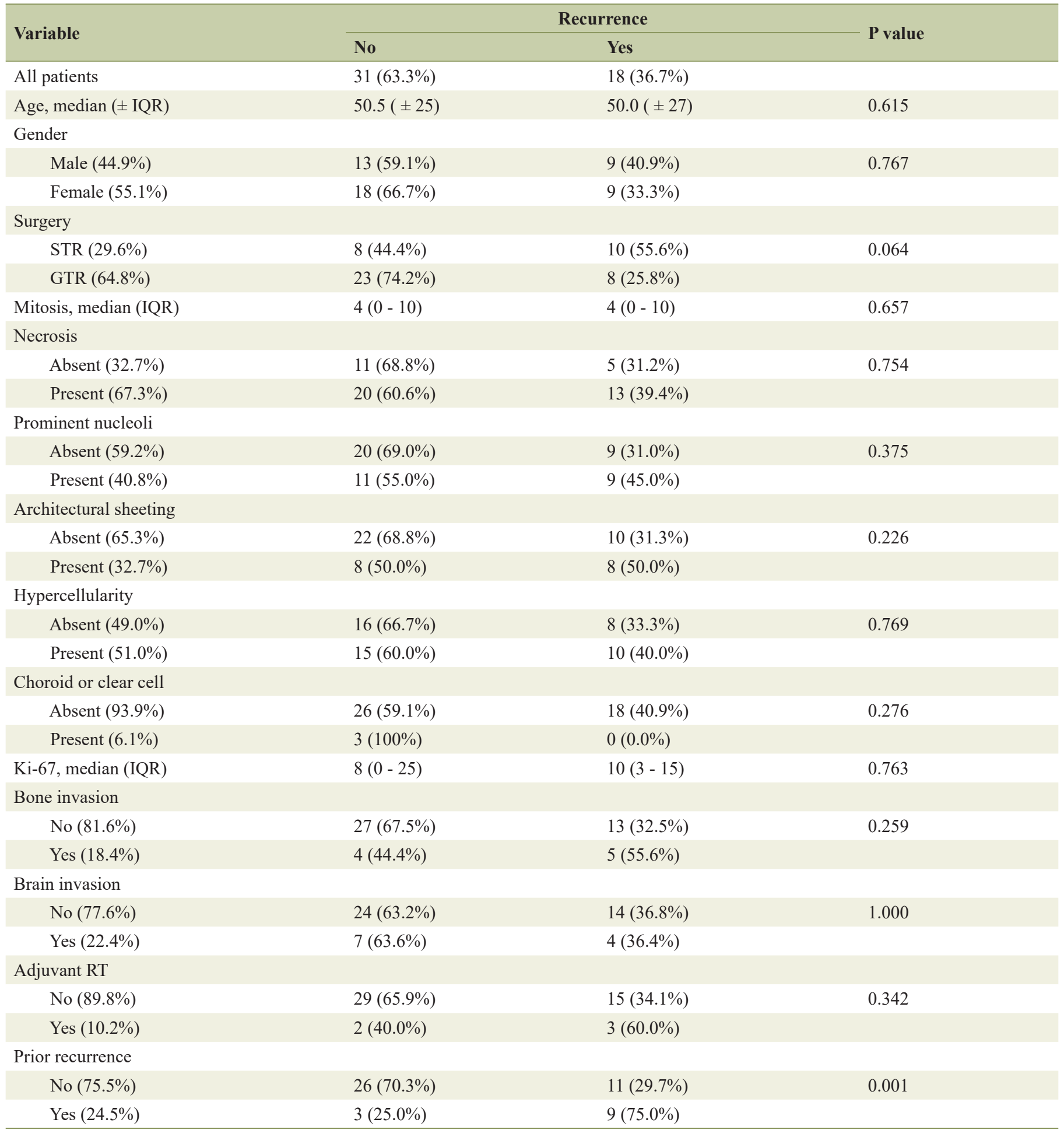

STR: subtotal resection; GTR: gross total resection; IQR: interquartile range; RT: radiation therapy.

ure in our analysis (Table 1). Upon further inspection using Cox proportional hazards $(\mathrm{CPH})$ modeling, extent of surgical resection $(\mathrm{P}=0.02)$ and the presence of prior recurrence $(\mathrm{P}$ $=0.002$ ) were strongly associated with time to recurrence on univariate analysis. On multivariable analysis, however, only prior recurrence remained associated with time to subsequent local failure (Table 2).

Of the 18 patients who suffered a local recurrence after 

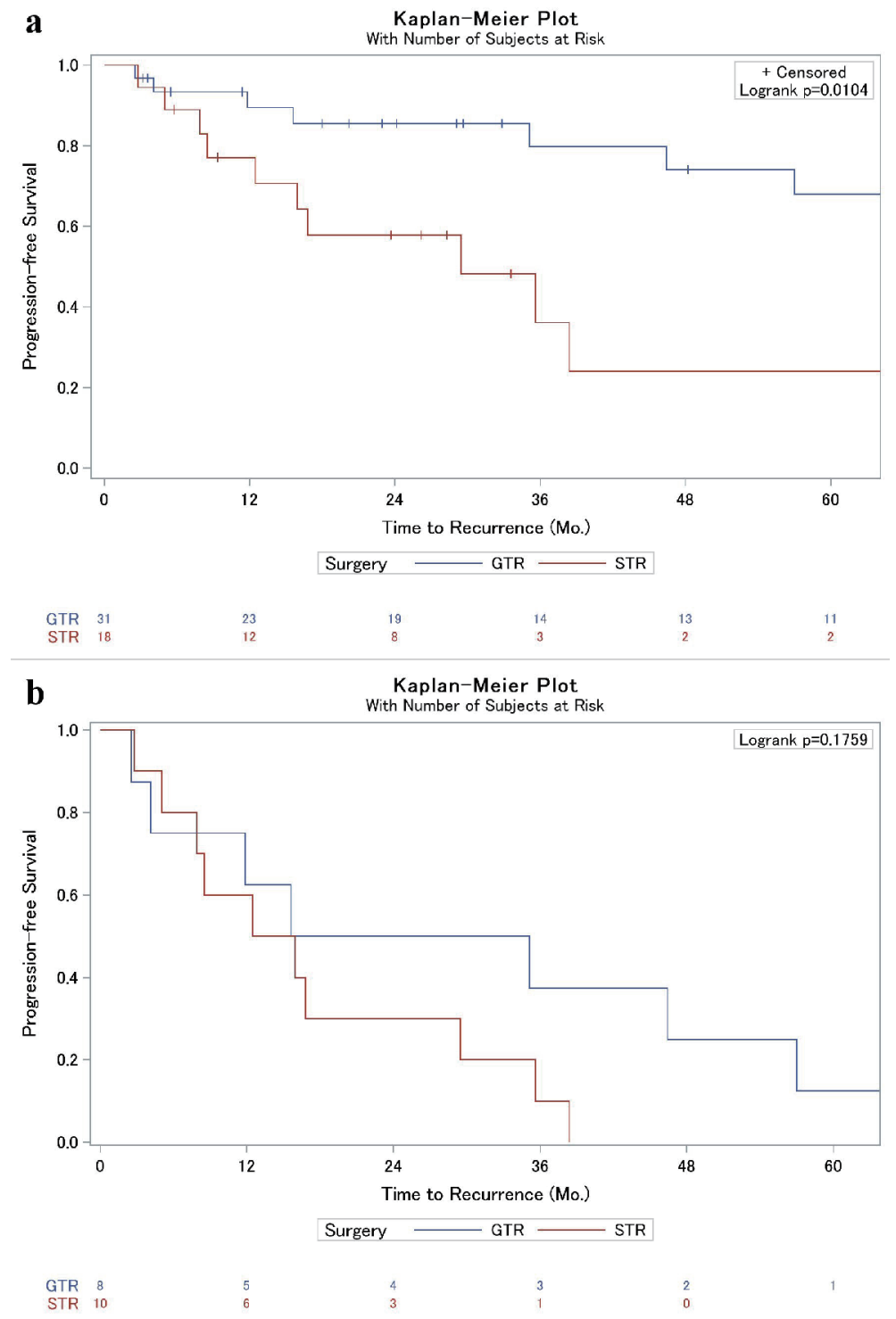

Figure 1. (a) Kaplan-Meier analysis of local failure of grade II meningiomas based on extent of surgical resection. (b) KaplanMeier sub-analysis of patients who suffered post-operative local failure (from Fig. 1a) to illustrate time to local failure difference between GTR and STR. Overall, progression was similar between the two cohorts $(P=0.18)$, but a sub-analysis after 12 months revealed a trend towards significance $(P=0.09)$. GTR: gross total resection, STR: subtotal resection.

surgical resection at our institution, eight patients (44.4\%) underwent salvage RT alone, four $(22.2 \%)$ underwent salvage resection alone, three (16.7\%) underwent resection followed by RT, one $(5.6 \%)$ underwent treatment with systemic therapy, and two $(11.1 \%)$ sought treatment at other institutions and were thus lost to follow-up. Despite these salvage therapies, 12 of these patients $(66.7 \%)$ suffered further subsequent tumor rerecurrence. Bone invasion on the initiation surgical resection was the only histologic variable associated with subsequent recurrence (Table 3). Interestingly, the only factor part of the patient's salvage regimen independently associated with the development of a second recurrence was the lack of salvage RT (Table 3). In fact, median progression-free survival was 45 months in those who received RT at the first recurrence vs. 4 months who did not receive RT (Fig. 3).

\section{Discussion}

The aim of our study was to determine which features of grade II meningiomas are predictive of recurrence, with the ultimate goal being to determine which patients may benefit most from adjuvant RT. Based on these findings, only the extent of surgical resection and local recurrence prior to being treated at our institute were predictive of post-operative recurrence. No studied histologic criteria were associated with risk of recurrence. Interestingly, in those who recurred following treatment at our university, only a lack of RT used during salvage and bone 


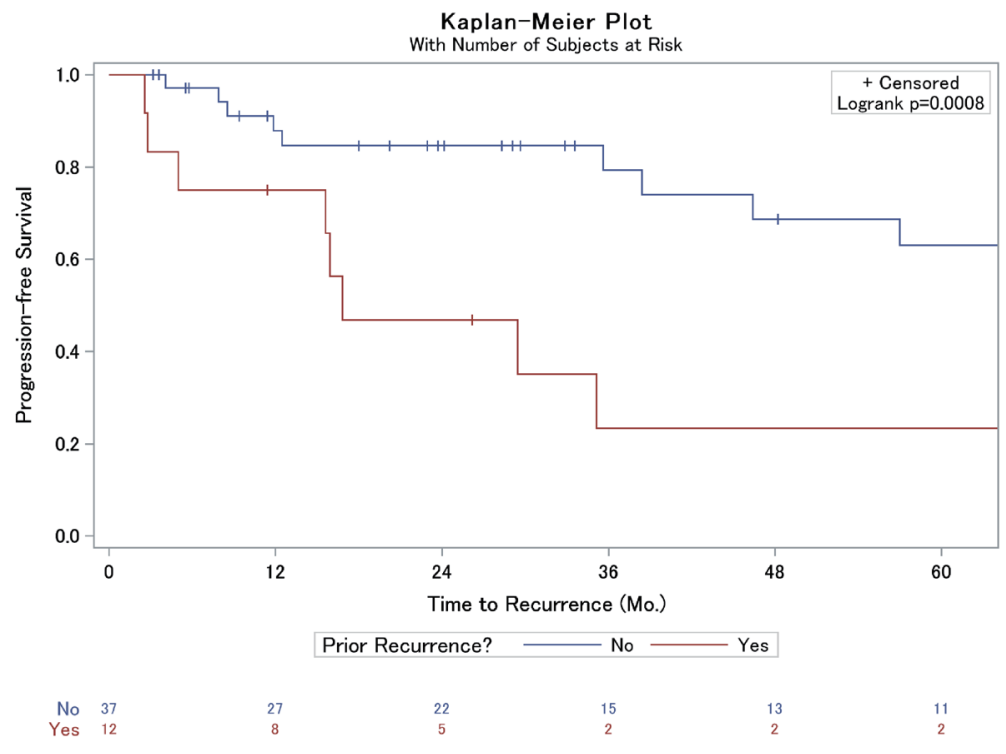

Figure 2. Kaplan-Meier analysis of local failure of grade II meningiomas based on prior meningioma recurrence.

invasion were associated with further recurrence.

This data suggest that the currently accepted histologic features used to determine tumor aggressiveness, and thus likelihood of local failure, poorly predict patient outcomes. Similar to the current study, past works have also failed to consistently establish associations between accepted histologic criteria and local recurrence in grade II meningiomas. Work by Barrett et al has previously suggested that the presence of necrosis was not predictive of recurrence in grade II meningiomas following GTR [8]. Further, studies attempting to associate Ki-67 with recurrence have been mixed. Nanda et al found that a $\mathrm{Ki}-67$ of less than $15 \%$ was associated with improved survival and associated with less risk of local recurrence in grade II meningiomas [4]. Barrett et al found local recurrence rates of $21 \%$ if $\mathrm{Ki}-67$ was $<10 \%$ but as high as $41 \%$ if $\mathrm{Ki}-67$ was $>10 \%$ [8]. Additionally, Champeaux and Dunn also found

Table 2. Factors Associated With Time to Recurrence Following Resection in Grade II Meningiomas

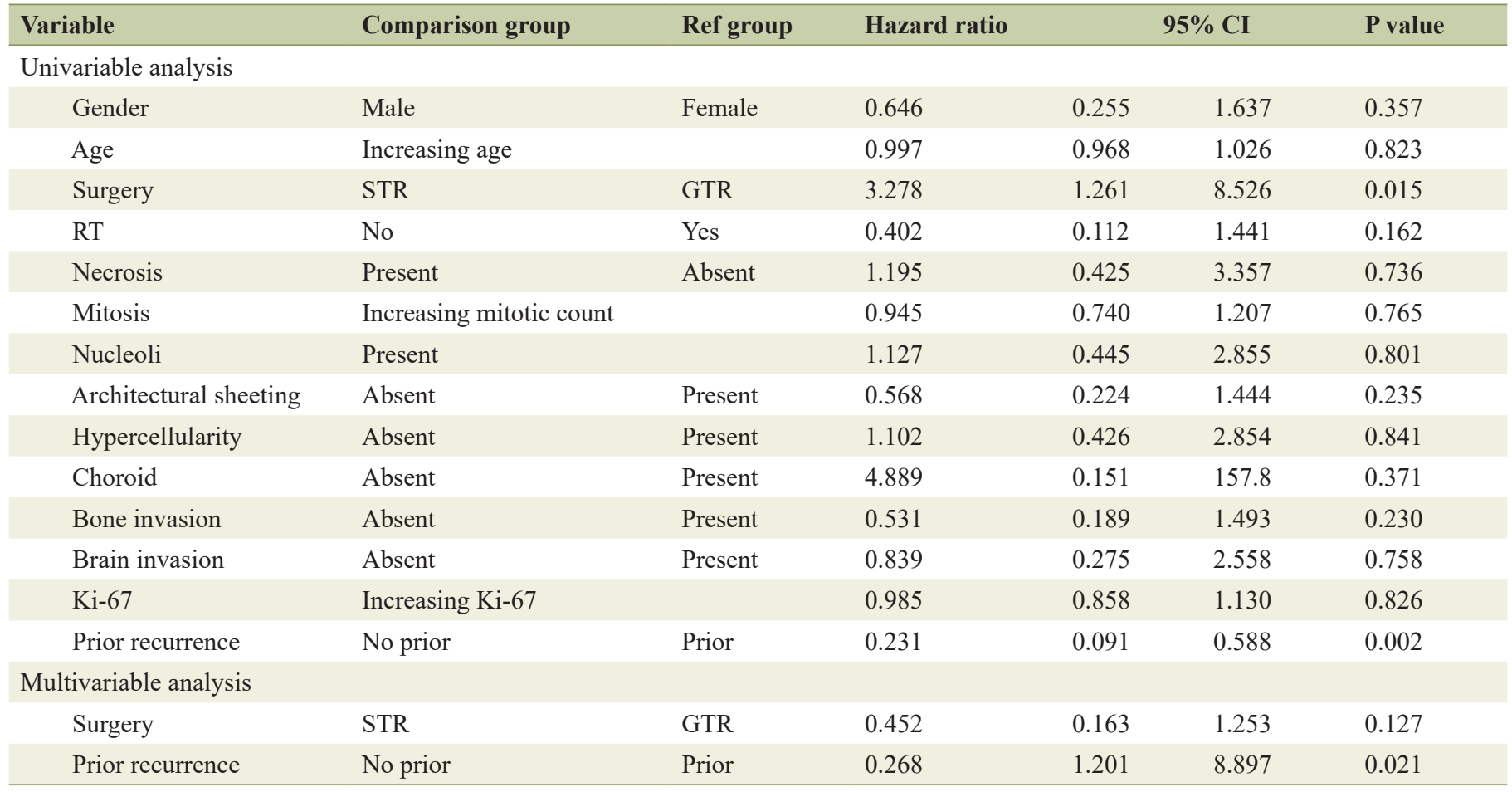

STR: subtotal resection; GTR: gross total resection; RT: radiation therapy; Cl: confidence interval. 
Table 3. Factors Associated With Time to Second Recurrence in Grade II Meningiomas

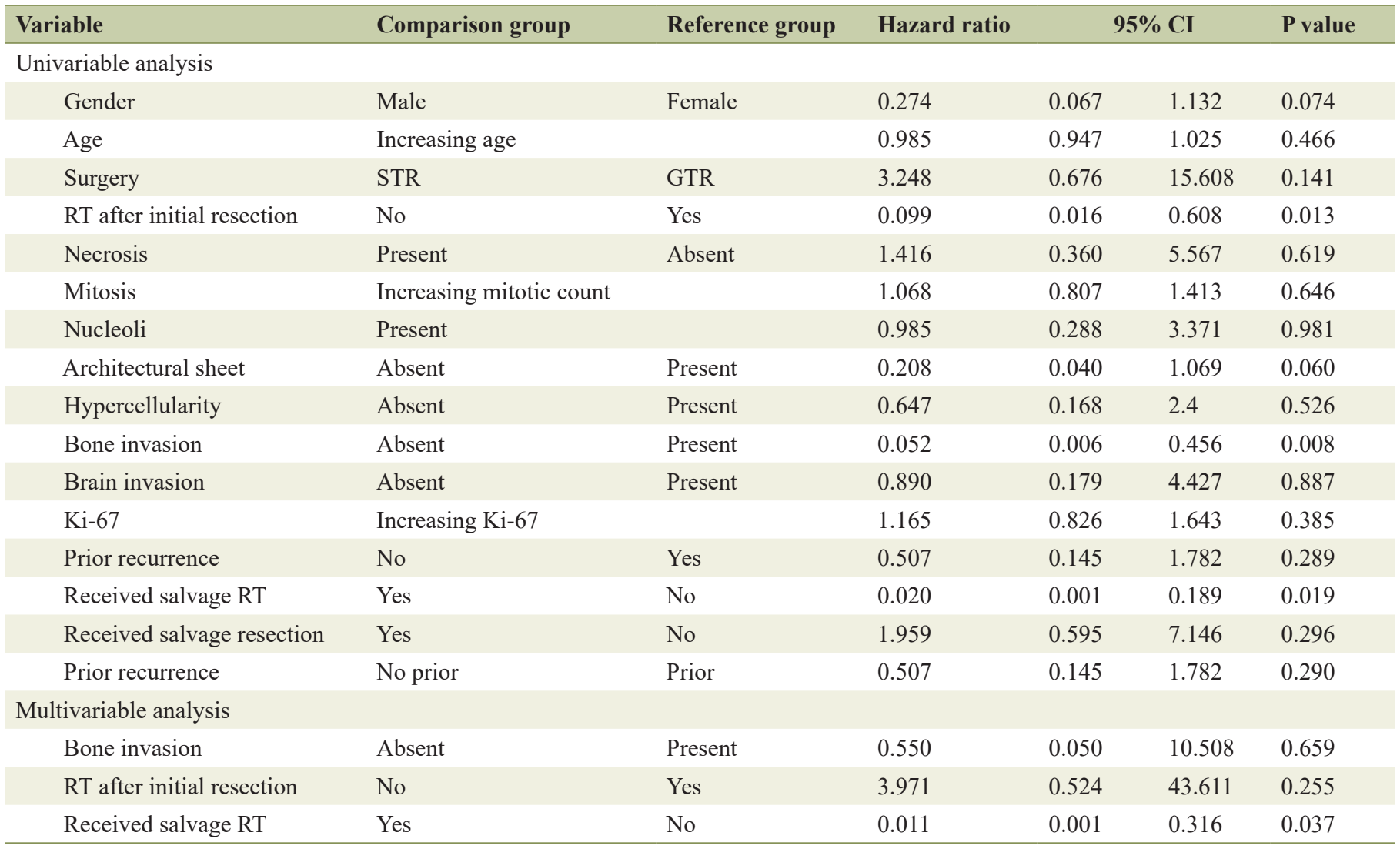

STR: subtotal resection; GTR: gross total resection; RT: radiation therapy; Cl: confidence interval.

that Ki-67 index predicted surgical recurrence-free survival [9]. However, as in the present study, Durand et al was not able to support Ki-67 as a prognostic factor and Jo et al and Aghi et al did not find $\mathrm{Ki}-67$ predictive of progression-free survival or overall survival [10-12]. Together, this data continue to indicate the need to establish more robust histologic criteria to predict aggressiveness of grade II meningiomas.

The prognostic implications of obtaining a GTR vs. STR

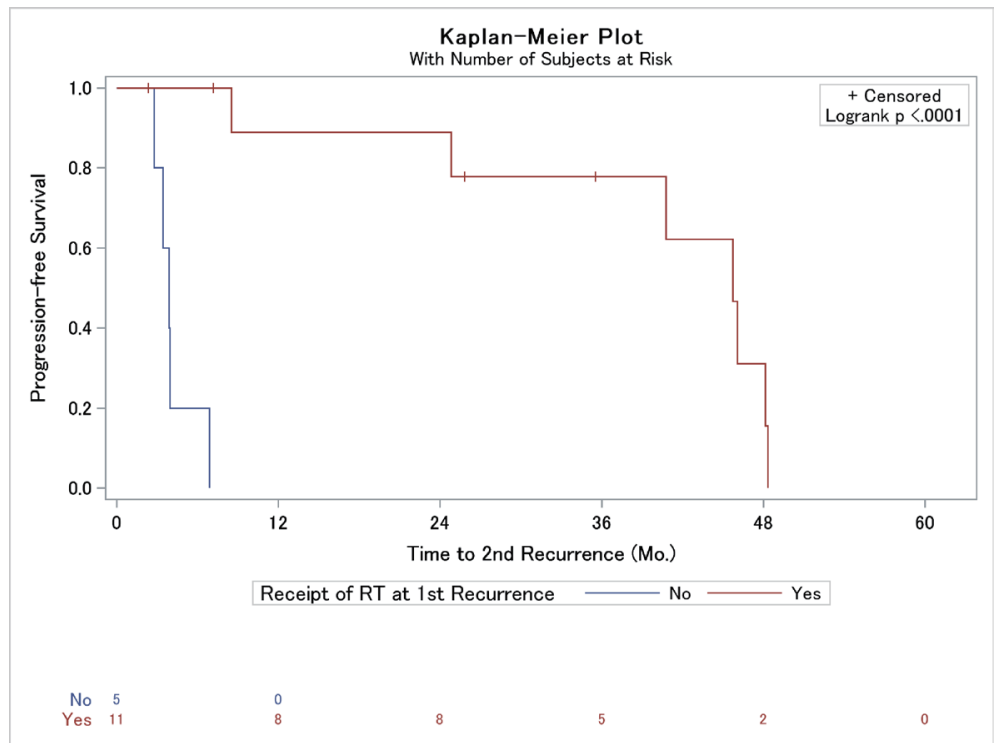

Figure 3. Kaplan-Meier analysis of the association of use of radiation therapy (RT) as a portion of post-recurrence patient sal- 
are well-established. Extent of resection has been studied by multiple groups previously, usually in the context of Simpson and Shinshu grade. Nanda et al found that the recurrence rate in Simpson grades I and II (GTR) was 31\%, but in grades III and IV (STR), recurrences more than doubled to $73 \%$. They found a similar trend using the Shinshu grading system [4]. Liu et al found the hazard ratio for local recurrence following STR to be 3.1 when compared to GTR [7]. Champeaux and Dunn also found that extent of resection was significantly associated with recurrence-free survival [9]. Together, these works strongly suggest that further therapy is most likely to be required in patients who undergo STR for a grade II meningioma. Further, the significant association of prior recurrence with subsequent local failure and the fact that only salvage RT was associated with reduced risk of further recurrence add further credence to the concept that patients with recurrent grade II meningiomas require further treatment to prevent local failure regardless of extent of surgical resection. Importantly, both of these patient populations were included in the high-risk arm of RTOG 0539 and received adjuvant RT to a dose of $60 \mathrm{~Gy}$, with this present data implying that such treatment may truly be necessary.

Overall, this study suggests that patients with past local failure or STR should be considered for adjuvant RT. These findings also provide evidence to suggest that after tumor recurrence for a grade II meningioma, forfeiting re-resection of the recurrent tumor if asymptomatic or with unsalvageable damage and offering salvage RT, could be a reasonable treatment option. This study further supports the idea to advocate for adjuvant RT for grade II meningiomas immediately after surgery even after GTR. In doing so, this may maximize the chances of local control over an observation approach followed by salvage RT, which appears to have a high likelihood of repetitive recurrences in the salvage situation. Currently, the NRG BN003 trial is ongoing to help delineate the best approach. So far, no clearly defined risk factors have been reported that can further stratify the risk levels of recurrence in WHO grade II meningiomas, pending the results from NRG BN003 trial. However, it is interesting to note that in our results, bone invasion, although not associated with recurrence after the initial surgery, was associated with time to the second failure, which is the first time that this is being reported to the best of our knowledge, and warrants further study.

There are several potential weaknesses to our study. The study was retrospective in nature and therefore could be subject to bias. Our patient number was also somewhat limited at 49. There were also inconsistencies in pathologic reporting (such as Ki-67), potentially blinding us to the effects these features had on local recurrence. A large number of patients in this study were referred to our institute by other providers after failing initial treatment. Thus, this may bias our patient population into a higher-risk population with more refractory/ aggressive disease. Additionally, the present study represents a single institution experience with several patients receiving pre-treatment at outside institutions for which some demographic (such as smoking status or body mass index (BMI)), operative and histologic details were not available. A larger database that is multi-institutional with consistent histologic and radiologic reporting may help elucidate which factors are predictive. Moreover, there has been a major recent trend to incorporate molecular parameters in tumor classifications, which are not available in our database.

\section{Conclusions}

History of past local failure and extent of surgical resection may be important factors in predicting recurrence in grade II meningioma. Patients with past local failure or subtotal resection should be considered for adjuvant RT. Currently considered histopathologic factors appear to possess little prognostic utility and may not be helpful in guiding adjuvant treatment.

\section{Acknowledgments}

None to declare.

\section{Financial Disclosure}

The authors certify that they have no affiliations with or involvement in any organization or entity with any financial interest (such as honoraria; educational grants; participation in speakers' bureaus; membership, employment, consultancies, stock ownership, or other equity interest; and expert testimony or patent-licensing arrangements), or non-financial interest (such as personal or professional relationships, affiliations, knowledge or beliefs) in the subject matter or materials discussed in this manuscript.

\section{Conflict of Interest}

The authors all declare that conflicts of interest do not exist.

\section{Informed Consent}

Not applicable.

\section{Author Contributions}

Drs. Baine, Sleightholm, and Zhang oversaw the project in every capacity from conception to finish. LZ, RS, MB were involved in the collection of the data. Drs. Baine and Sleightholm were involved in the statistical analysis. All authors were involved in the drafting and final approval of the text, tables, figures and manuscript as a whole.

\section{Data Availability}

Any inquiries regarding supporting data availability of this study should be directed to the corresponding author. 


\section{References}

1. Mawrin C, Perry A. Pathological classification and molecular genetics of meningiomas. J Neurooncol. 2010;99(3): 379-391.

2. Saraf S, McCarthy BJ, Villano JL. Update on meningiomas. Oncologist. 2011;16(11):1604-1613.

3. Lubgan D, Rutzner S, Lambrecht U, Rossler K, Buchfelder M, Eyupoglu I, Fietkau R, et al. Stereotactic radiotherapy as primary definitive or postoperative treatment of intracranial meningioma of WHO grade II and III leads to better disease control than stereotactic radiotherapy of recurrent meningioma. J Neurooncol. 2017;134(2):407416.

4. Nanda A, Bir SC, Konar S, Maiti T, Kalakoti P, Jacobsohn JA, Guthikonda B. Outcome of resection of WHO Grade II meningioma and correlation of pathological and radiological predictive factors for recurrence. J Clin Neurosci. 2016;31:112-121.

5. Przybylowski CJ, Raper DM, Starke RM, Xu Z, Liu KC, Sheehan JP. Stereotactic radiosurgery of meningiomas following resection: predictors of progression. J Clin Neurosci. 2015;22(1):161-165.

6. Rogers L, Zhang P, Vogelbaum MA, Perry A, Ashby LS, Modi JM, Alleman AM, et al. Intermediate-risk meningioma: initial outcomes from NRG Oncology RTOG 0539.
J Neurosurg. 2018;129(1):35-47.

7. Liu X, Shan B, Wang M, Xu J. World Health Organization Grade II Meningiomas: the role of adjuvant/salvage gamma knife surgery after initial surgery and prognostic factor assessment. World Neurosurg. 2018;109:e352e362.

8. Barrett OC, Hackney JR, McDonald AM, Willey CD, Bredel M, Fiveash JB. Pathologic predictors of local recurrence in atypical meningiomas following gross total resection. Int J Radiat Oncol Biol Phys. 2019;103(2):453-459.

9. Champeaux C, Dunn L. World Health Organization Grade II Meningioma: a 10-year retrospective study for recurrence and prognostic factor assessment. World Neurosurg. 2016;89:180-186.

10. Durand A, Labrousse F, Jouvet A, Bauchet L, Kalamarides M, Menei P, Deruty R, et al. WHO grade II and III meningiomas: a study of prognostic factors. J Neurooncol. 2009;95(3):367-375.

11. Jo K, Park HJ, Nam DH, Lee JI, Kong DS, Park K, Kim JH. Treatment of atypical meningioma. J Clin Neurosci. 2010;17(11):1362-1366.

12. Aghi MK, Carter BS, Cosgrove GR, Ojemann RG, AminHanjani S, Martuza RL, Curry WT, Jr., et al. Long-term recurrence rates of atypical meningiomas after gross total resection with or without postoperative adjuvant radiation. Neurosurgery. 2009;64(1):56-60; discussion 60. 